\title{
REGULARIZATION AND BOUNDARY CONDITIONS FOR THE 13 MOMENT EQUATIONS
}

\author{
HENNING STRUCHTRUP \\ ETH Zürich, Department of Materials, Polymer Physics, CH-8093 Zürich, \\ Switzerland (on leave from University of Victoria, Canada, struchtr@uvic.ca) \\ MANUEL TORRILHON \\ ETH Zürich, Seminar for Applied Mathematics, CH-8092 Zürich, Switzerland
}

\begin{abstract}
We summarize our recent contributions to the development of macroscopic transport equations for rarefied gas flows. A combination of the ChapmanEnskog expansion and Grad's moment method, termed as the order of magnitude method, yields the regularized 13 moment equations (R13 equations) which are of super-Burnett order. A complete set of boundary conditions is derived from the boundary conditions of the Boltzmann equations. The R13 equations are linearly stable and their results for Knudsen numbers below 0.5 stand in excellent agreement to DSMC simulations.
\end{abstract}

\section{Introduction}

Processes in rarefied gases are well described by the Boltzmann equation $[1,2]$ which describes the evolution of the particle distribution function in phase space, i.e. on the microscopic level.

The relevant scaling parameter to characterize processes in rarefied gases is the Knudsen number Kn, defined as the ratio between the mean free path of a particle and a relevant length scale. If the Knudsen number is small, the Boltzmann equation can be reduced to simpler models, which allow faster solutions. Indeed, if $\mathrm{Kn}<0.01$ (say), the hydrodynamic equations, the laws of Navier-Stokes and Fourier (NSF), can be derived from the Boltzmann equation, e.g. by the Chapman-Enskog method $[1,2]$. The NSF equations are macroscopic equations for mass density $\rho$, velocity $v_{i}$ and temperature $T$, and thus pose a mathematically less complex problem than the Boltzmann equation.

Macroscopic equations for rarefied gas flows at Knudsen numbers above 0.01 promise to replace the Boltzmann equation with simpler equations 
that still capture the relevant physics. The Chapman-Enskog expansion is the classical method to achieve this goal, but the resulting Burnett and super-Burnett equations are unstable [3].

A classical alternative is Grad's moment method [4] which extends the set of variables by adding deviatoric stress tensor $\sigma_{i j}$, heat flux $q_{i}$, and possibly higher moments of the phase density. The resulting equations are stable but lead to spurious discontinuities in shocks, and for a given value of the Knudsen number it is not clear what set of moments one would have to consider [2].

Struchtrup and Torrilhon combined both approaches by performing a Chapman-Enskog expansion around a non-equilibrium phase density of Grad type [5,6] which resulted in the "regularized 13 moment equations" (R13 equations) which form a stable set of equations for the 13 variables $\left(\rho, v_{i}, T, \sigma_{i j} . q_{i}\right)$ of super-Burnett order.

An alternative approach to the problem was presented by Struchtrup in $[7,8]$, partly based on earlier work by Müller et al. [9]. The Order of Magnitude Method, which is briefly outlined in Section 2, is based on a rigorous asymptotic analysis of the infinite hierarchy of the moment equations.

One of the biggest problems for all models beyond NSF is to prescribe suitable boundary conditions for the extended equations, which should follow from the boundary conditions for the Boltzmann equation. This task was recently tackled in [11], and our solution to the problem [12] will be briefly discussed in Section 3, which presents boundary conditions for the R13 equations.

Section 4 will briefly discuss the properties of the R13 equations, which are linearly stable, obey a H-theorem for the linear case, contain the Burnett and super-Burnett equations asymptotically, predict phase speeds and damping of ultrasound waves in excellent agreement to experiments, yield smooth and accurate shock structures for all Mach numbers, and exhibit Knudsen boundary layers and the Knudsen minimum in excellent agreement to DSMC simulations.

Lack of space forbids to present any details, the interested reader is referred to the cited literature, including the monograph [2].

\section{The Order of Magnitude Method}

The Order of Magnitude Method [7,8] considers not the Boltzmann equation itself, but its infinite system of moment equations. The method of finding the proper equations with order of accuracy $\lambda_{0}$ in the Knudsen number consists of the following three steps: 
(1) Determination of the order of magnitude $\lambda$ of the moments.

(2) Construction of moment set with minimum number of moments at order $\lambda$.

(3) Deletion of all terms in all equations that would lead only to contributions of orders $\lambda>\lambda_{0}$ in the conservation laws for energy and momentum.

Step 1 is based on a Chapman-Enskog expansion where a moment $\phi$ is expanded according to $\phi=\phi_{0}+\mathrm{Kn} \phi_{1}+\mathrm{Kn}^{2} \phi_{2}+\mathrm{Kn}^{3} \phi_{3}+\cdots$, and the leading order of $\phi$ is determined by inserting this ansatz into the complete set of moment equations. A moment is said to be of leading order $\lambda$ if $\phi_{\beta}=0$ for all ${ }^{\beta}<\lambda$. This first step agrees with the ideas of [9]. Alternatively, the order of magnitude of the moments can be found from the principle that a single term in an equation cannot be larger in size by one or several orders of magnitude than all other terms [10].

In Step 2, new variables are introduced by linear combination of the moments originally chosen. The new variables are constructed such that the number of moments at a given order $\lambda$ is minimal. This step gives an unambiguous set of moments at order $\lambda$.

Step 3 follows from the definition of the order of accuracy $\lambda_{0}$ : A set of equations is said to be accurate of order $\lambda_{0}$, when stress and heat flux are known within the order $\mathcal{O}\left(\mathrm{Kn}^{\lambda_{0}}\right)$.

The order of magnitude method gives the Euler and NSF equations at zeroth and first order, and thus agrees with the Chapman-Enskog method in the lower orders [7]. The second order equations turn out to be Grad's 13 moment equations for Maxwell molecules [7], and a generalization of these for molecules that interact with power potentials $[2,8]$. At third order, the method was only performed for Maxwell molecules, where it yields the R13 equations [7], which read ( $\theta$ is the temperature in energy units, $\mu$ is the viscosity of the gas)

$$
\begin{gathered}
\frac{D \rho}{D t}+\rho \frac{\partial v_{k}}{\partial x_{k}}=0 \\
\rho \frac{D v_{i}}{D t}+\rho \frac{\partial \theta}{\partial x_{i}}+\theta \frac{\partial \rho}{\partial x_{i}}+\frac{\partial \sigma_{i k}}{\partial x_{k}}=0 \\
\frac{3}{2} \rho \frac{D \theta}{D t}+\rho \theta \frac{\partial v_{k}}{\partial x_{k}}+\frac{\partial q_{k}}{\partial x_{k}}+\sigma_{k l} \frac{\partial v_{k}}{\partial x_{l}}=0 \\
\frac{D \sigma_{i j}}{D t}+\frac{4}{5} \frac{\partial q_{\langle i}}{\partial x_{j\rangle}}+2 \sigma_{k\langle i} \frac{\partial v_{j\rangle}}{\partial x_{k}}+\sigma_{i j} \frac{\partial v_{k}}{\partial x_{k}}+\frac{\partial u_{i j k}^{0}}{\partial x_{k}}=-\rho \theta\left[\frac{\sigma_{i j}}{\mu}+2 \frac{\partial v_{\langle i}}{\partial x_{j\rangle}}\right]
\end{gathered}
$$


4

$$
\begin{array}{r}
\frac{D q_{i}}{D t}+\frac{5}{2} \sigma_{i k} \frac{\partial \theta}{\partial x_{k}}-\sigma_{i k} \theta \frac{\partial \ln \rho}{\partial x_{k}}+\theta \frac{\partial \sigma_{i k}}{\partial x_{k}}+\frac{7}{5} q_{i} \frac{\partial v_{k}}{\partial x_{k}}+\frac{7}{5} q_{k} \frac{\partial v_{i}}{\partial x_{k}}+\frac{2}{5} q_{k} \frac{\partial v_{k}}{\partial x_{i}} \\
-\frac{\sigma_{i j}}{\varrho} \frac{\partial \sigma_{j k}}{\partial x_{k}}+\frac{1}{2} \frac{\partial w_{i k}^{1}}{\partial x_{k}}+\frac{1}{6} \frac{\partial w^{2}}{\partial x_{i}}+u_{i j k}^{0} \frac{\partial v_{j}}{\partial x_{k}}=-\frac{5}{2} \rho \theta\left[\frac{q_{i}}{\kappa}+\frac{\partial \theta}{\partial x_{i}}\right] \\
w^{2}=-\frac{\sigma_{i j} \sigma_{i j}}{\rho}-12 \frac{\mu}{p}\left[\theta \frac{\partial q_{k}}{\partial x_{k}}+\frac{5}{2} q_{k} \frac{\partial \theta}{\partial x_{k}}-\theta q_{k} \frac{\partial \ln \rho}{\partial x_{k}}+\theta \sigma_{i j} \frac{\partial v_{i}}{\partial x_{k}}\right] \\
u_{i j k}^{0}=-2 \frac{\mu}{p}\left[\theta \frac{\partial \sigma_{\langle i j}}{\partial x_{k\rangle}}-\sigma_{\langle i j} \frac{\partial \ln \rho}{\partial x_{k\rangle}}+\frac{4}{5} q_{\langle i} \frac{\partial v_{j}}{\partial x_{k\rangle}}\right] \\
w_{i j}^{1}=-\frac{4}{7} \frac{\sigma_{k\langle i} \sigma_{j\rangle k}}{\rho}-\frac{24}{5} \frac{\mu}{p}\left[\theta \frac{\partial q_{\langle i}}{\partial x_{j\rangle}}+q_{\langle i} \frac{\partial \theta}{\partial x_{j\rangle}}-\theta q_{\langle i} \frac{\partial \ln \rho}{\partial x_{j\rangle}}\right. \\
\left.+\frac{5}{7} \theta\left(\sigma_{k\langle i} \frac{\partial v_{j\rangle}}{\partial x_{k}}+\sigma_{k\langle i} \frac{\partial v_{k}}{\partial x_{j\rangle}}-\frac{2}{3} \sigma_{i j} \frac{\partial v_{k}}{\partial x_{k}}\right)\right]
\end{array}
$$

\section{Boundary Conditions for R13}

The computation of boundary conditions for the R13 equations is based on Maxwell's model for boundary conditions for the Boltzmann equation [1,2], which states that a fraction $\chi$ of the particles hitting the wall is thermalized, while the remaining $1-\chi$ particles are specularly reflected. Boundary conditions for moments follow by taking moments of the boundary conditions of the Boltzmann equation. To produce meaningful boundary conditions, one needs to obey the following rules:

(1) Continuity: In order to have meaningful boundary conditions for all accommodation coefficients $\chi$ in $[0,1]$, only boundary conditions for tensors with an odd number of normal components should be considered $[3,11,12]$.

(2) Consistency: Only boundary conditions for fluxes that actually appear in the equations should be considered [12].

(3) Coherence: The same number of boundary conditions should be prescribed for the linearized and the non-linear equations [12].

The application of Rules 1 and 2 is straightforward and yields the following set of boundary conditions ( $t$ and $n$ denote tangential and normal tensor components, respectively, and $V_{t}=v_{t}-v_{t}^{W}, \beta=\frac{\chi}{2-\chi} \sqrt{\frac{2}{\pi \theta}}, P=$ $\left.\rho \theta+\frac{1}{2} \sigma_{n n}-\frac{1}{28} \frac{w_{n n}}{\theta}-\frac{1}{120} \frac{w_{k k}}{\theta}\right)$

$$
\sigma_{n t}=-\beta\left[P V_{t}+\frac{1}{5} q_{t}+\frac{1}{2} u_{t n n}^{0}\right]
$$




$$
\begin{aligned}
w_{t n} & =\beta\left[P \theta V_{t}-\frac{1}{2} \theta u_{t n n}^{0}-\frac{11}{5} \theta q_{t}-P V_{t}^{3}+6 P\left(\theta-\theta_{W}\right) V_{t}\right] \\
q_{n} & =-\beta\left[2 P\left(\theta-\theta_{W}\right)+\frac{5}{28} w_{n n}+\frac{1}{15} w_{k k}+\frac{1}{2} \theta \sigma_{n n}-\frac{1}{2} P V_{t}^{2}\right] \\
u_{n n n}^{0} & =\beta\left[\frac{2}{5} P\left(\theta-\theta_{W}\right)-\frac{1}{14} w_{n n}+\frac{1}{75} w_{k k}-\frac{7}{5} \sigma_{n n}-\frac{3}{5} P V_{t}^{2}\right] \\
u_{t t n}^{0}+\frac{1}{2} u_{n n n}^{0} & =-\beta\left[\theta\left(\sigma_{t t}+\frac{1}{2} \sigma_{n n}\right)+\frac{1}{14}\left(w_{t t}+\frac{1}{2} w_{n n}\right)-\frac{1}{2} P V_{t}^{2}\right]
\end{aligned}
$$

$\theta_{W}$ and $v^{W}$ denote temperature and velocity of the wall. The first condition above is the slip condition for the velocity, while the third equation is the jump condition for the temperature. In a manner of speaking, the other conditions can be described as jump conditions for higher moments.

When the R13 equations are considered for channel flows in their original form, it turns out that a different number of boundary conditions is required to solve the fully non-linear and the linearized equations. Since this would not allow a smooth transition between linear and non-linear situations, we formulated the third rule as given above.

Asymptotic analysis shows that some terms can be changed without changing the overall asymptotic accuracy of the R13 equations. This leads to the algebraization of several non-linear terms in the pde's which, after some algebra, leads to algebraic relations, termed as bulk equations, between the moments which serve as additional boundary conditions for the non-linear equations [12],

$$
w_{n n}=\frac{136}{25} \frac{q_{n}^{2}}{p}-\frac{72}{35} \frac{\sigma_{t n}^{2}}{\rho} \quad, \quad u_{t n n}^{0}=\frac{32}{45} \frac{\sigma_{t n} q_{n}}{p}
$$

\section{Computations and Simulations}

We summarize the most important features of the R13 equations which result from analytical considerations, and from analytical and numerical solutions:

The R13 equations:

- are derived in a rational manner by means of the order of magnitude method $[7,8]$, or from a Chapman-Enskog expansion around nonequilibrium $[5,6]$,

- are of third order in the Knudsen number [2,5-8],

- are linearly stable for initial and boundary value problems $[5,6]$,

- contain Burnett and super-Burnett asymptotically $[5,6]$, 
- predict phase speeds and damping of ultrasound waves in excellent agreement to experiments [5],

- give smooth shock structures for all Mach numbers, with good agreement to DSMC simulations for $\mathrm{Ma}<3$ [6],

- are accompanied by a complete set of boundary conditions [12],

- obey an H-theorem for the linear case, including the boundaries [13],

- exhibit the Knudsen paradox for channel flows [12,13],

- exhibit Knudsen boundary layers in good agreement to DSMC [14,15],

- are accessible to numerical simulations in higher dimensions [16],

- predict light scattering spectra in accordance to experiments [17]

With these properties and features, the R13 equations must be considered as the most successful macroscopic model for rarefied gas flows. The application of the R13 equations to a wider variety of one-, two, and three-dimensional rarefied gas flow problems is planned for the future.

Acknowledgments: H.S.: Support by the Natural Sciences and Engineering Research Council (NSERC) is gratefully acknowledged. I like to thank Prof. H.C. Öttinger (ETH Zürich) for his kind hospitality. M.T.: Support through the EURYI award of the European Science Foundation (ESF) is gratefully acknowledged.

\section{References}

1. C. Cercignani, The Boltzmann Equation and its Applications, Applied Mathematical Sciences 67, Springer, New York 1988

2. H. Struchtrup, Macroscopic Transport Equations for Rarefied Gas FlowsApproximation Methods in Kinetic Theory, Springer, Heidelberg 2005

3. A.V. Bobylev, Sov. Phys. Dokl. 27, 29 (1982)

4. H. Grad, Comm. Pure Appl. Math. 2, 331 (1949)

5. H. Struchtrup and M. Torrilhon, Phys. Fluids 15, 2668 (2003)

6. M. Torrilhon and H. Struchtrup, J. Fluid Mech. 513, 171 (2004)

7. H. Struchtrup, Phys. Fluids 16, 3921 (2004)

8. H. Struchtrup, Multiscale Model. Simul. 3, 221 (2005)

9. I. Müller, D. Reitebuch, W. Weiss, Cont. Mech. Thermodyn. 15, 113 (2003)

10. H. Struchtrup, J. Stat. Phys. 125, 565 (2006)

11. X. Gu and D. Emerson, J. Comput. Phys. 225, 263 (2007)

12. M. Torrilhon and H. Struchtrup, submitted to J. Comp. Phys. (2007)

13. H. Struchtrup and M. Torrilhon, Phys. Rev. Lett. 99, 014502 (2007)

14. H. Struchtrup, Cont. Mech. Thermodyn. 17, 43-50 (2005)

15. H. Struchtrup and T. Thatcher, Cont. Mech. Thermodyn. 19, 177 (2007)

16. M. Torrilhon, Multiscale Model. Sim. 5, 695 (2006)

17. M. Torrilhon, Proceedings 25th Intl. Symposium on Rarefied Gas Dynamics, in press (2007) 